\title{
Space-Time Block Coding with Beamforming for Triple-Polarized Uniform Linear Array Systems
}

\author{
Xin Su${ }^{1}$ and Kyung Hi Chang ${ }^{2}$ \\ ${ }^{1}$ The Program in IT \& Media Convergence Studies, Inha University, Incheon 402-751, Republic of Korea \\ ${ }^{2}$ The Electronic Engineering Department, Inha University, Incheon 402-751, Republic of Korea \\ Correspondence should be addressed to Kyung Hi Chang; khchang@inha.ac.kr
}

Received 25 May 2015; Revised 8 August 2015; Accepted 20 August 2015

Academic Editor: Mourad Nedil

Copyright (c) $2015 \mathrm{X}$. Su and K. H. Chang. This is an open access article distributed under the Creative Commons Attribution License, which permits unrestricted use, distribution, and reproduction in any medium, provided the original work is properly cited.

Generally, space-time block coding (STBC) and beamforming (BF) gains cannot be obtained simultaneously because the former performs well under a low correlated MIMO channel, and the latter works efficiently in an environment with high correlation. However, array systems with antenna polarization have the potential to achieve gains with both techniques simultaneously because the cross-branch links in the system are usually uncorrelated. The cross-array links, on the other hand, can be highly correlated by setting the array element space equal to, or less than, a half-wavelength. This paper proposes a scheme to explore STBC and BF simultaneously via a triple-polarized uniform linear array (TPULA) system. The proposed scheme was verified based on the Long Term Evolution-Advanced (LTE-A) specification under a polarized MIMO (PMIMO) channel model, and therewith, the simulation results confirmed the validity of our proposed scheme.

\section{Introduction}

Recently, there has been a gradual demand for the use of a polarized antenna system, especially for $5 \mathrm{G}$ networks. This is mainly because antenna polarization is a crucial resource to be exploited for the design of space-limited wireless devices. Techniques such as space-time diversity, multiplexing, and array processing can be applied to polarized antenna systems to boost system throughput. Although many research articles and ongoing projects have been working on antenna polarization [1-3], exploration into using space-time block coding (STBC) and beamforming (BF) techniques for polarized antenna systems has not received much attention in the literature. Typically, there is no efficient approach to simultaneously obtaining STBC and BF gains in polarized antenna systems. This paper proposes a robust scheme to cope with this constraint by applying STBC in three transmit antennae and $\mathrm{BF}$ techniques via a triple-polarized uniform linear array (TPULA) system. That is, the polarized antenna branches are grouped into three sets that steer three orthogonal beams at the $X-Y, Z-X$, and $Y-Z$ planes. STBC is additionally applied at three orthogonally colocated branches of each array element (AE) to accommodate the space-time diversity gain.
Via the proposed scheme by exploring the characteristics of antenna polarization, the MIMO techniques, that is, STBC and BF, can be realized at mobile stations (MSs) simultaneously to guarantee the system performance. For example, two AEs could be employed at an MS with a space of $8 \mathrm{~cm}$ when the carrier frequency is $2 \mathrm{GHz}$. At each $\mathrm{AE}$, three orthogonally colocated antenna branches perform STBC that can further enhance the system performance. The implementation complexity is increased by the proposed scheme that costs higher power consumption for the MS because it needs to calculate the $\mathrm{BF}$ weights as well as performing the STBC decoding. However, it is very pivotal for celledge MSs to keep the performance in 5G communication systems, especially for the applications without tolerance of retransmissions. In the simulation results, the block error rate (BLER) performance improves, which shows the validity of our proposed scheme.

The remainder of this paper is organized as follows: Section 2 provides the TPULA configuration and Section 3 describes our proposed scheme. The simulation results are discussed in Section 4, and finally, our conclusions are drawn in Section 5. 


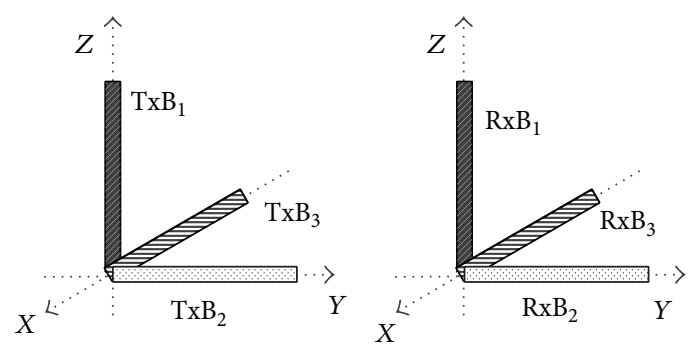

(a) $\mathrm{TPMIMO}_{\mathrm{B}}$.
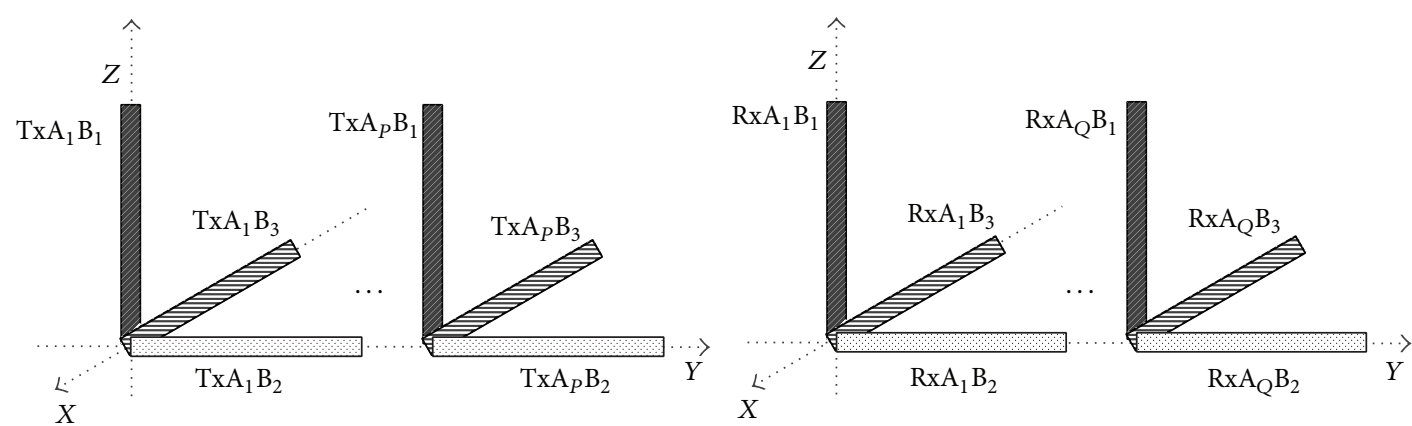

(b) TPMIMO $_{\mathrm{A \& B}}$ (TPULA)

FIGURE 1: Orthogonal TPMIMO system.

\section{TPULA Configuration}

Figure 1 provides examples of orthogonal triple-polarized MIMO (TPMIMO) system in the array and branch (A\&B) multiple antennae configuration. According to Figure 1(a), there are three orthogonally colocated antenna branches at the transmitter $(\mathrm{Tx})$ and receiver $(\mathrm{Rx})$ ports. Therefore, this PMIMO transceiver is defined at the branch level as $\mathrm{TPMIMO}_{\mathrm{B}}$. By uniformly aligning the polarized antenna ports (AEs) at the Tx and the Rx, as illustrated in Figure 1(b), a PMIMO system is defined as TPMIMO ${ }_{\mathrm{A} \& \mathrm{~B}}$, which indicates that antenna polarization is realized at both branch and array levels.

The beamwidth is relevant to the array configuration, where it is inversely proportional to the number of AEs and the AE space. Because the space among three colocated branches at each AE is fixed at zero, as shown in Figure 1, which makes beamwidth scale up to $360^{\circ}$, the beams should be generated via corresponding cross-array branches rather than the colocated branches at each AE [3]. Therefore, a TPULA system can generate three orthogonal beams as follows:

(i) beam steered and varied in the $X-Y$ plane is formed by the set of branches of $T / \operatorname{RxA}_{p(q)} \mathrm{B}_{1}$, where $p(q)=$ $1,2, \ldots, P(Q)$;

(ii) beam steered and varied in the $Z$ - $X$ plane is formed by the set of branches of $T / \operatorname{RxA}_{p(q)} \mathrm{B}_{2}$;

(iii) beam steered and varied in the $Y-Z$ plane is formed by the set of branches of $T / \operatorname{RxA}_{p(q)} \mathrm{B}_{3}$.

Figure 2 depicts the 3D beams generated by a TPULA system, where three orthogonal beams can be steered and varied separately on the $X-Y, Z-X$, and $Y-Z$ planes. By applying the TPULA into an orthogonal frequency division multiplexing (OFDM) system, considering STBC and BF, a robust MIMO transceiver is provided, as seen in Figure 3, which is explained in detail in the following section.

\section{TPULA System with STBC and Beamforming}

As studied by [1], the cross-array links (e.g., from $\mathrm{TxA}_{1} \mathrm{~B}_{1}$ to $\mathrm{RxA}_{1} \mathrm{~B}_{1}$ and $\mathrm{RxA}_{2} \mathrm{~B}_{1}$ ) are highly correlated by setting the $\mathrm{AE}$ space equal to, or less than, a half-wavelength. The cross-branch links (e.g., from $\mathrm{TxA}_{1} \mathrm{~B}_{1}$ and $\mathrm{TxA}_{1} \mathrm{~B}_{2}$ to $\mathrm{RxA}_{1} \mathrm{~B}_{1}$ ), however, are usually uncorrelated due to the space polarization. This inspired us to incorporate STBC and BF techniques simultaneously in the TPULA system to boost performance. Moreover, in order to achieve full-rate coding with an odd number of transmit antennae, quasiorthogonal STBC (QO-STBC) has emerged in the literature [4, 5], which fully explores diversity gain but increases the complexity of decoding due to nonorthogonal interference. In this paper, we propose a scheme to combine the QO-STBC for three transmit antennae with BF techniques via a TPULA system as follows.

In Figure 3, the BF weights $\left(w_{p_{b}}^{\mathrm{Tx}}\right.$ and $\left.w_{q_{b}}^{\mathrm{Rx}}\right)$ are multiplied before the inverse fast Fourier transform (IFFT) block of the Tx and after the FFT block of the $\mathrm{Rx}$, where $p(1,2, \ldots P)$, $q(1,2, \ldots Q)$, and $b(1,2,3)$ denote the indexes of the TxAE, RxAE, and antenna branch, respectively. Note that we have considered the digital $\mathrm{BF}$, where the TxBF weights obtained by zero-forcing $\mathrm{BF}$ are determined by the $\mathrm{Rx}$ location and the RxBF weights obtained by MMSE BF are determined based 

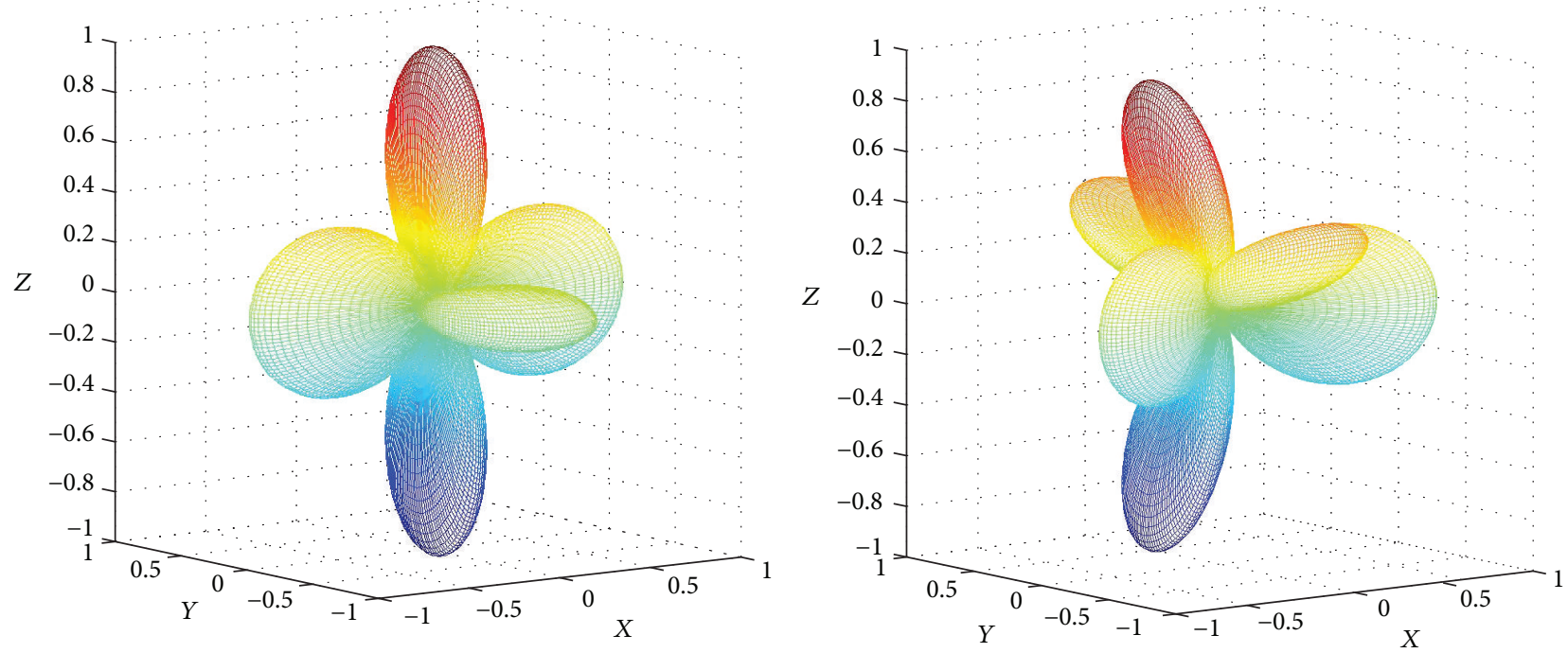

FIgURE 2: 3D beams generated by a TPULA system.

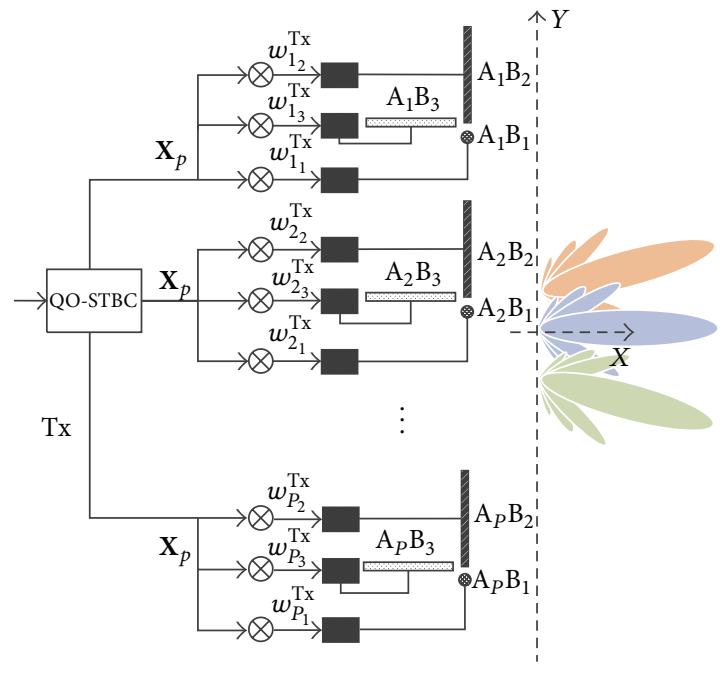

IFFT and GI extension

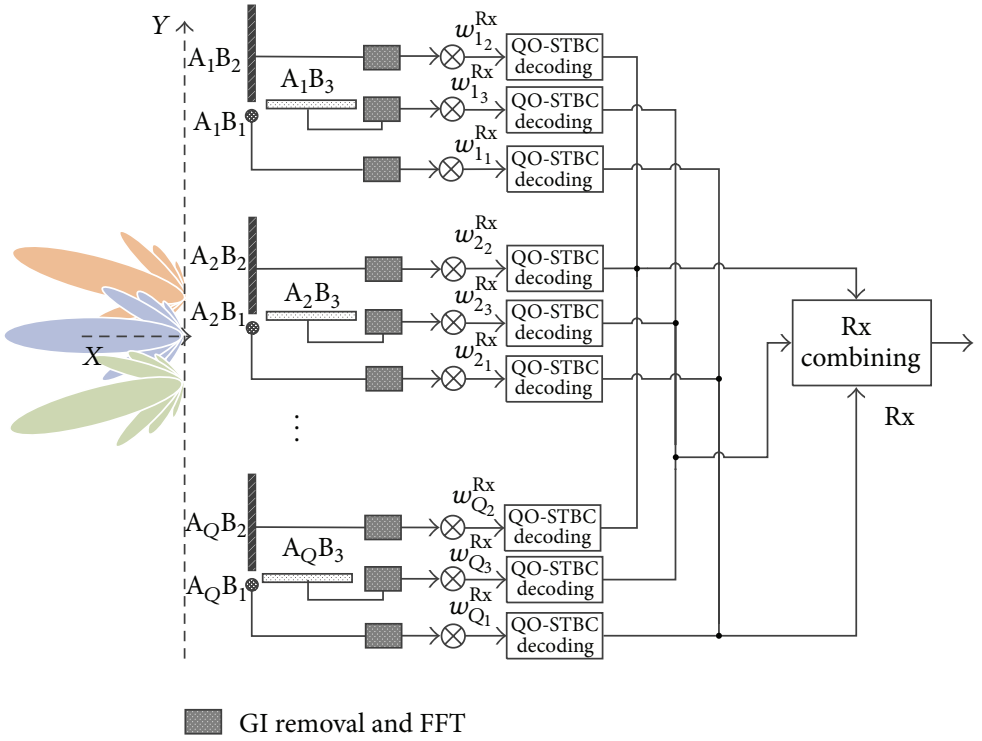

GI removal and FFT

Figure 3: OFDM-based TPULA system with STBC and BF.

on reference signal. The BF weights then differentiate each other based on the polarized antenna branches of each array element instead of each subcarrier. $\mathbf{X}_{p}$ in expression (1) is the transmitted QO-STBC symbol matrix [4], where the Roman numeral is the symbol index, $i$ is the time index modulo of 4 $(I=4)$, and $j$ is the antenna index modulo of $3(J=3)$ :

$$
\mathbf{X}_{p}=\left(\begin{array}{ccc}
x_{\mathrm{II}} & x_{\mathrm{III}} & x_{\mathrm{IV}} \\
x_{\mathrm{I}}^{*} & -x_{\mathrm{IV}}^{*} & x_{\mathrm{III}}^{*} \\
x_{\mathrm{IV}} & x_{\mathrm{I}} & x_{\mathrm{II}} \\
x_{\mathrm{III}}^{*} & -x_{\mathrm{II}}^{*} & x_{\mathrm{I}}^{*}
\end{array}\right)_{I-b y-J} .
$$

For the cross-branch links from the $p$ th TxAE with three branches to a single $\mathrm{Rx}$ branch $q_{b}$, that is, $\mathbf{h}_{p \sim q_{b}}$, the received signal before the RxBF weights multiplication is given as

$$
\begin{aligned}
\mathbf{R}_{p \sim q_{b}} & =\left(\mathbf{h}_{p \sim q_{b}}\left(\mathbf{W}_{p}^{\mathrm{Tx}} \odot \mathbf{X}_{p}\right)^{T}\right)^{T}+\mathbf{n}_{p \sim q_{b}} \\
& =\left(\mathbf{W}_{p}^{\mathrm{Tx}} \odot \mathbf{X}_{p}\right) \mathbf{h}_{p \sim q_{b}}^{T}+\mathbf{n}_{p \sim q_{b}} \\
& =\left(\begin{array}{llll}
r_{p \sim q_{b}, \mathrm{I}} & r_{p \sim q_{b}, \mathrm{II}} & r_{p \sim q_{b}, \mathrm{III}} & r_{p \sim q_{b}, \mathrm{IV}}
\end{array}\right)^{T},
\end{aligned}
$$


where "๑" denotes the Hadamard product and $\mathbf{W}_{p}^{\mathrm{Tx}}$ is the TxBF weighting matrix given by

$$
\mathbf{W}_{p}^{\mathrm{Tx}}=\left(\begin{array}{ccc}
w_{p_{1}}^{\mathrm{Tx}} & w_{p_{2}}^{\mathrm{Tx}} & w_{p_{3}}^{\mathrm{Tx}} \\
w_{p_{1}}^{\mathrm{Tx}} & w_{p_{2}}^{\mathrm{Tx}} & w_{p_{3}}^{\mathrm{Tx}} \\
w_{p_{1}}^{\mathrm{Tx}} & w_{p_{2}}^{\mathrm{Tx}} & w_{p_{3}}^{\mathrm{Tx}} \\
w_{p_{1}}^{\mathrm{Tx}} & w_{p_{2}}^{\mathrm{Tx}} & w_{p_{3}}^{\mathrm{Tx}}
\end{array}\right) .
$$

$\mathbf{h}_{p \sim q_{b}}$ is the vector of the polarized cross-branch links, given as

$$
\mathbf{h}_{p \sim q_{b}}=\left(\begin{array}{lll}
h_{p_{1} \sim q_{b}} & h_{p_{2} \sim q_{b}} & h_{p_{3} \sim q_{b}}
\end{array}\right),
$$

and $\mathbf{n}_{p \sim q_{b}}$ is the noise vector given by

$$
\mathbf{n}_{p \sim q_{b}}=\left(\begin{array}{llll}
\sigma_{p \sim q_{b}, \mathrm{I}} & \sigma_{p \sim q_{b}, \mathrm{II}} & \sigma_{p \sim q_{b}, \mathrm{III}} & \sigma_{p \sim q_{b}, \mathrm{IV}}
\end{array}\right)^{T} .
$$

The minimum mean square error (MMSE) RxBF is used to minimize the error between the RxBF output and the available reference signal, where the optimal weights $w_{q_{b}}^{\mathrm{Rx}}$ can be obtained by the Wiener-Hoff equation [6]. Then, the signal after RxBF is given by

$$
\widetilde{\mathbf{R}}_{p \sim q_{b}}=w_{q_{b}}^{\mathrm{Rx}} \mathbf{R}_{p \sim q_{b}} .
$$

The STBC decoding applied after the RxBF gives

$$
\begin{aligned}
\widetilde{\mathbf{X}}_{p \sim q_{b}} & =\mathbf{D}_{p \sim q_{b}} \mathbf{T}_{p \sim q_{b}} \\
& =\left(\begin{array}{llll}
\tilde{x}_{p \sim q_{b}, \mathrm{I}} & \tilde{x}_{p \sim q_{b}, \mathrm{II}} & \tilde{x}_{p \sim q_{b}, \mathrm{III}} & \tilde{x}_{p \sim q_{b}, \mathrm{IV}}
\end{array}\right)^{T} .
\end{aligned}
$$

Here, $\mathbf{D}_{p \sim q_{b}}$ is the STBC decoding matrix, which is modified based on equation (12) in [4] by considering TxBF weights given by

$$
\begin{aligned}
& \mathbf{D}_{p \sim q_{b}} \\
& =\left(\begin{array}{cccc}
0 & w_{p_{1}}^{\mathrm{Tx}} h_{p_{1} \sim q_{b}} & \left(w_{p_{2}}^{\mathrm{Tx}}\right)^{*} h_{p_{2} \sim q_{b}}^{*} & w_{p_{3}}^{\mathrm{TX}} h_{p_{3} \sim q_{b}} \\
\left(w_{p_{1}}^{\mathrm{Tx}}\right)^{*} h_{p_{1} \sim q_{b}}^{*} & 0 & \left(w_{p_{3}}^{\mathrm{Tx}}\right)^{*} h_{p_{3} \sim q_{b}}^{*} & -w_{p_{2}}^{\mathrm{T} \mathrm{x}} h_{p_{2} \sim q_{b}} \\
\left(w_{p_{2}}^{\mathrm{Tx}}\right)^{*} h_{p_{p^{\prime}} \sim q_{b}}^{*} & w_{p_{3}}^{\mathrm{Tx}} h_{p_{3} \sim q_{b}} & 0 & w_{p_{1}}^{\mathrm{Tx}} h_{p_{1} \sim q_{b}} \\
\left(w_{p_{3}}^{\mathrm{Tx}}\right)^{*} h_{p_{3} \sim q_{b}}^{*} & -w_{p_{2}}^{\mathrm{TX}} h_{p_{2} \sim q_{b}} & \left(w_{p_{1}}^{\mathrm{Tx}}\right)^{*} h_{p_{1} \sim q_{b}}^{*} & 0
\end{array}\right) .
\end{aligned}
$$

And $\mathbf{T}_{p \sim q_{b}}$ is defined as

$$
\mathbf{T}_{p \sim q_{b}}=\left(\begin{array}{llll}
\widetilde{r}_{p \sim q_{b}, \mathrm{I}} & \widetilde{r}_{p \sim q_{b}, \mathrm{II}}^{*} & \widetilde{r}_{p \sim q_{b}, \mathrm{III}} & \widetilde{r}_{p \sim q_{b}, \mathrm{IV}}^{*}
\end{array}\right)^{T}
$$

by taking the conjugate of the second and the fourth elements of $\widetilde{\mathbf{R}}_{p \sim q_{b}}$. The final output of the proposed scheme is obtained by the last functional block in Figure 3 (i.e., the Rx combining), given as

$$
\widetilde{\mathbf{X}}=\sum_{q=1}^{Q} \sum_{b=1}^{3}\left(\begin{array}{llll}
\tilde{x}_{p \sim q_{b}, \mathrm{I}} & \tilde{x}_{p \sim q_{b}, \mathrm{II}} & \tilde{x}_{p \sim q_{b}, \mathrm{III}} & \tilde{x}_{p \sim q_{b}, \mathrm{IV}}
\end{array}\right)^{T} .
$$

Li et al. [4] used the receiver reported by Liu et al. [7] to cope with the interference caused by nonorthogonality of the QO-STBS. In this paper, the complexity of interference cancellation can be simplified by the adaptive setting of TxBF weights in (3). By calculation, the decoded signal in (10) is given by

$$
\begin{aligned}
& \tilde{x}_{p \sim q_{b}, \mathrm{I}}=\underbrace{\left(w_{q_{b}}^{\mathrm{Rx}}\right)^{2}\left(\left|w_{p_{1}}^{\mathrm{Tx}} h_{p_{1} \sim q_{b}}\right|^{2}+\left|w_{p_{2}}^{\mathrm{Tx}} h_{p_{2} \sim q_{b}}\right|^{2}+\left|w_{p_{3}}^{\mathrm{Tx}} h_{p_{3} \sim q_{b}}\right|^{2}\right) x_{\mathrm{I}}}_{\text {Desired singal }} \\
& +\underbrace{\left(w_{q_{b}}^{\mathrm{Rx}}\right)^{2}\left[w_{p_{1}}^{\mathrm{Tx}} h_{p_{1} \sim q_{b}}\left(w_{p_{3}}^{\mathrm{Tx}}\right)^{*} h_{p_{3} \sim q_{b}}^{*}+w_{p_{3}}^{\mathrm{Tx}} h_{p_{p} \sim q_{b}}\left(w_{p_{1}}^{\mathrm{Tx}}\right)^{*} h_{p_{1} \sim q_{b}}^{*}\right] x_{\mathrm{III}}}_{\text {Interference caused by adjacent signal }} \\
& +\underbrace{\left(w_{q_{b}}^{\mathrm{Rx}}\right)^{2}\left[w_{p_{1}}^{\mathrm{Tx}} h_{p_{1} \sim q_{b}} \sigma_{p \sim q_{b}, \mathrm{II}}^{*}+\left(w_{p_{2}}^{\mathrm{Tx}}\right)^{*} h_{p_{2} \sim q_{b}}^{*} \sigma_{p \sim q_{b}, \mathrm{III}}+w_{p_{3}}^{\mathrm{Tx}} h_{p_{3} \sim q_{b}} \sigma_{p \sim q_{b}, \mathrm{IV}}^{*}\right]}_{\text {Noise }}, \\
& \tilde{x}_{p \sim q_{b}, \mathrm{II}}=\left(w_{q_{b}}^{\mathrm{Rx}}\right)^{2}\left(\left|w_{p_{1}}^{\mathrm{Tx}} h_{p_{1} \sim q_{b}}\right|^{2}+\left|w_{p_{2}}^{\mathrm{Tx}} h_{p_{2} \sim q_{b}}\right|^{2}+\left|w_{p_{3}}^{\mathrm{Tx}} h_{p_{3} \sim q_{b}}\right|^{2}\right) x_{\mathrm{II}} \\
& +\left(w_{q_{b}}^{\mathrm{Rx}}\right)^{2}\left[w_{p_{1}}^{\mathrm{Tx}} h_{p_{1} \sim q_{b}}\left(w_{p_{3}}^{\mathrm{Tx}}\right)^{*} h_{p_{3} \sim q_{b}}^{*}+w_{p_{3}}^{\mathrm{Tx}} h_{p_{3} \sim q_{b}}\left(w_{p_{1}}^{\mathrm{Tx}}\right)^{*} h_{p_{1} \sim q_{b}}^{*}\right] x_{\mathrm{IV}} \\
& +\left(w_{q_{b}}^{\mathrm{Rx}}\right)^{2}\left[w_{p_{1}}^{\mathrm{Tx}} h_{p_{1} \sim q_{b}} \sigma_{p \sim q_{b}, \mathrm{II}}^{*}+\left(w_{p_{2}}^{\mathrm{Tx}}\right)^{*} h_{p_{2} \sim q_{b}}^{*} \sigma_{p \sim q_{b}, \mathrm{III}}+w_{p_{3}}^{\mathrm{Tx}} h_{p_{3} \sim q_{b}} \sigma_{p \sim q_{b}, \mathrm{IV}}^{*}\right], \\
& \tilde{x}_{p \sim q_{b}, \mathrm{III}}=\left(w_{q_{b}}^{\mathrm{Rx}}\right)^{2}\left(\left|w_{p_{1}}^{\mathrm{Tx}} h_{p_{1} \sim q_{b}}\right|^{2}+\left|w_{p_{2}}^{\mathrm{Tx}} h_{p_{2} \sim q_{b}}\right|^{2}+\left|w_{p_{3}}^{\mathrm{Tx}} h_{p_{3} \sim q_{b}}\right|^{2}\right) x_{\mathrm{III}} \\
& +\left(w_{q_{b}}^{\mathrm{Rx}}\right)^{2}\left[w_{p_{1}}^{\mathrm{Tx}} h_{p_{1} \sim q_{b}}\left(w_{p_{3}}^{\mathrm{Tx}}\right)^{*} h_{p_{3} \sim q_{b}}^{*}+w_{p_{3}}^{\mathrm{Tx}} h_{p_{3} \sim q_{b}}\left(w_{p_{1}}^{\mathrm{Tx}}\right)^{*} h_{p_{1} \sim q_{b}}^{*}\right] x_{\mathrm{I}} \\
& +\left(w_{q_{b}}^{\mathrm{Rx}}\right)^{2}\left[w_{p_{1}}^{\mathrm{Tx}} h_{p_{1} \sim q_{b}} \sigma_{p \sim q_{b}, \mathrm{II}}^{*}+\left(w_{p_{2}}^{\mathrm{Tx}}\right)^{*} h_{p_{2} \sim q_{b}}^{*} \sigma_{p \sim q_{b}, \mathrm{III}}+w_{p_{3}}^{\mathrm{Tx}} h_{p_{3} \sim q_{b}} \sigma_{p \sim q_{b}, \mathrm{IV}}^{*}\right],
\end{aligned}
$$




$$
\begin{aligned}
\tilde{x}_{p \sim q_{b}, \mathrm{IV}}= & \left(w_{q_{b}}^{\mathrm{Rx}}\right)^{2}\left(\left|w_{p_{1}}^{\mathrm{Tx}} h_{p_{1} \sim q_{b}}\right|^{2}+\left|w_{p_{2}}^{\mathrm{Tx}} h_{p_{2} \sim q_{b}}\right|^{2}+\left|w_{p_{3}}^{\mathrm{Tx}} h_{p_{3} \sim q_{b}}\right|^{2}\right) x_{\mathrm{IV}} \\
& +\left(w_{q_{b}}^{\mathrm{Rx}}\right)^{2}\left[w_{p_{1}}^{\mathrm{Tx}} h_{p_{1} \sim q_{b}}\left(w_{p_{3}}^{\mathrm{Tx}}\right)^{*} h_{p_{3} \sim q_{b}}^{*}+w_{p_{3}}^{\mathrm{Tx}} h_{p_{3} \sim q_{b}}\left(w_{p_{1}}^{\mathrm{Tx}}\right)^{*} h_{p_{1} \sim q_{b}}^{*}\right] x_{\mathrm{II}} \\
& +\left(w_{q_{b}}^{\mathrm{Rx}}\right)^{2}\left[w_{p_{1}}^{\mathrm{Tx}} h_{p_{1} \sim q_{b}} \sigma_{p \sim q_{b}, \mathrm{II}}^{*}+\left(w_{p_{2}}^{\mathrm{Tx}}\right)^{*} h_{p_{2} \sim q_{b}}^{*} \sigma_{p \sim q_{b}, \mathrm{III}}+w_{p_{3}}^{\mathrm{Tx}} h_{p_{3} \sim q_{b}} \sigma_{p \sim q_{b}, \mathrm{IV}}^{*}\right] .
\end{aligned}
$$

Formulae (11) indicate that $x_{\mathrm{I}}$ and $x_{\mathrm{III}}$ interfere with each other, as well as $x_{\mathrm{II}}$ and $x_{\mathrm{IV}}$. For interference cancellation, it is better to set the scaling factor, that is, either $w_{p_{1}}^{\mathrm{Tx}}$ or $w_{p_{3}}^{\text {Tx }}$, to zero. The setting of zero needs to be determined by the off boresight angle (OBSA) effect discussed by Liu [8], where boresight refers to the axis vertical to the orientation of the array alignment. For example, in Figure 4, the solid beam is generated by the set of branches of $\operatorname{TxA}_{p} B_{1}$, while the dotted beam is generated by the set of branches of $\mathrm{TxA}_{p} \mathrm{~B}_{3}$. According to Liu [8], the beamwidth is known and is directly proportional to the OBSA value. Therefore, $w_{p_{3}}^{\mathrm{Tx}}=$ 0 (when $\alpha<\beta$ ) or $w_{p_{1}}^{\text {Tx }}=0$ (when $\alpha>\beta$ ) can be suggested to avoid increasing the beamwidth. Note that, in this paper, the location of the $\mathrm{Rx}$ is assumed to be known by the Tx. Additionally, by using the zero-forcing TxBF, we can have two options for TxBF weight settings, that is, $\left(w_{p_{1}}=0, w_{p_{2}}=\right.$ $\left.h_{p_{2}}^{-1}, w_{p_{3}}=h_{p_{3}}^{-1}\right)$ or $\left(w_{p_{3}}=0, w_{p_{2}}=h_{p_{2}}^{-1}, w_{p_{1}}=h_{p_{1}}^{-1}\right)$, which is determined mainly based on the location of the Rx. Note that we did not focus on the TDD system; therefore, the channel reciprocity is not performed. Since we assume the location of $\mathrm{Rx}$ is known by the Tx, the BF weight used in the paper is inverse of the array propagation factor at Tx side [3].

According to Figure 3, the proposed TPULA system can be easily extended as the polarized massive MIMO (PMMIMO) system by employing a large number of AEs, which requires a large number (tens or hundreds) of antennae serving at both Tx and Rx. Consequently, the system design then should consider the plenty of power consumption and high cost of RF chains. Antenna selection scheme is necessary for PM-MIMO system in both transmitting end and receiving end. An energy efficient antenna selection algorithm based on convex optimization might be proposed, where the number of transmit antenna and the number of receive antenna need to be jointly optimized to maximize energy efficiency. Good performance gain of energy efficiency should be obtained comparing with no antenna selection case.

\section{Performance Verification}

We verified the proposed scheme via Monte Carlo simulations based on the LTE-A specification, where Table 1 lists the details of the parameter settings. The downlink data mapping for LTE-A resource blocks used in the simulations is demonstrated in Figure 5, which is based on the QOSTBC symbol matrix of expression (1). $P_{\mathrm{A}_{p} \mathrm{~B}_{1}}, P_{\mathrm{A}_{p} \mathrm{~B}_{2}}$, and $P_{\mathrm{A}_{p} \mathrm{~B}_{3}}$ denote the pilots used for practical channel estimation, and a total of 24 pilots were used separately for 24 transmit antennae. An extension of work by Su et al. [9] is applied to simulations of the polarized MIMO channel, for which channel characteristics are also listed in Table 1. Note that the location of the $\mathrm{Rx}$ is updated periodically at each simulation loop.

Since we have proposed the scheme to combine STBC and BF firstly and could not find any existing combined STBC$\mathrm{BF}$ scheme for the performance comparison according to literatures, we only compared four transmission cases in this paper to verify our proposal by discussing the achievement of STBC and BF gains. Figure 6 shows the simulated BLER curves, including Case 1 of TPULA only (without STBC and BF), Case 2 of TPULA with STBC, Case 3 of TPULA with STBC and TxBF, and Case 4 of TPULA with STBC and TRxBF, where the target BLER for performance comparison is fixed at $10^{-2}$. According to Figure 6, Case 1 has the worst performance, because there is no space-time coding and BF gain achieved. By applying the receiver studied by Liu et al. [7] for interference cancellation, almost $1 \mathrm{~dB}$ of signal-to-noise ratio (SNR) gain at the target BLER can be achieved with Case 2 under the indoor environment, compared with Case 1. In addition, compared with Case 2, about $2.3 \mathrm{~dB}$ of SNR gain under the indoor environment at the target BLER is obtained with Case 3 when the TxBF is applied. Compared with Case 3, $0.5 \mathrm{~dB}$ SNR gain at the target BLER is achieved by using the MMSE RxBF via Case 4 because the amount of error fed into the STBC decoding process is further decreased. The error floors occur under the outdoor environment due to high mobility at $100 \mathrm{~km} / \mathrm{h}$. This indicates that the number of pilots in the TPULA system is not enough to compensate the channel correctly under an environment with fast timevarying phase response. Again, the efficiency of our proposed scheme can be quantified in terms of throughput. Figure 7 illustrates the simulated throughput curves. By focusing on the curves obtained under the indoor environment, we can observe that Case 4 of TPULA with STBC and TRxBF can achieve the highest throughput of $21 \mathrm{Mbps}$ at $E_{b} / N_{0}$ of $0 \mathrm{~dB}$ and $20 \mathrm{Mbps}, 19 \mathrm{Mbps}$, and $17 \mathrm{Mbps}$ for Case 3, Case 2, and Case 1 at $E_{b} / N_{0}$ of $0 \mathrm{~dB}$, respectively.

In addition, we evaluate the average BLER performance when two mobile users are employed. In this case, the eight AEs at Tx are divided into two groups, where each group is with four AEs and performs TxBF for a mobile user. Figure 8 illustrates the simulation results of average BLER for two users with the transmission Case 4. According to Figure 8, we can observe that the performance decreases a lot due to the loss of diversity order at Tx because multiusers need 


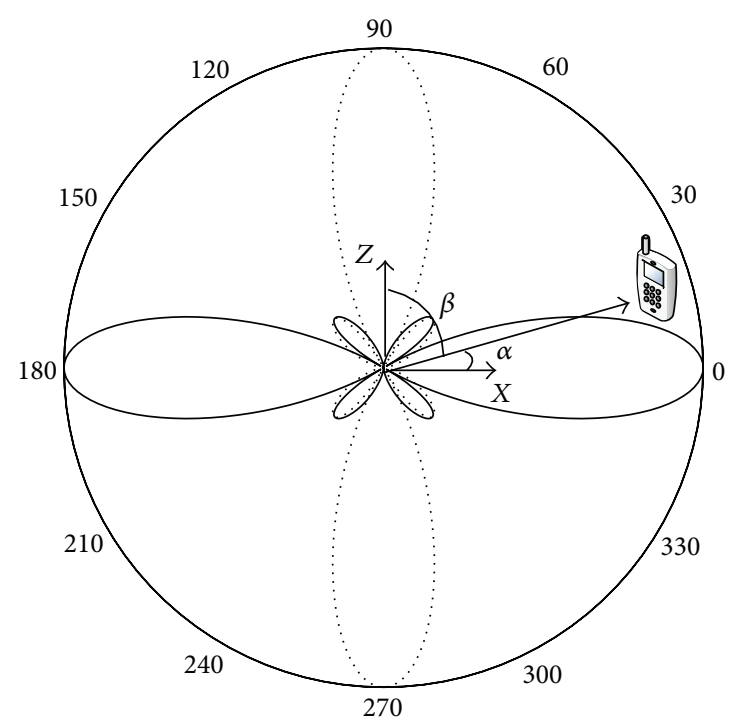

FIgURE 4: Setting of zero for TxBF weights.

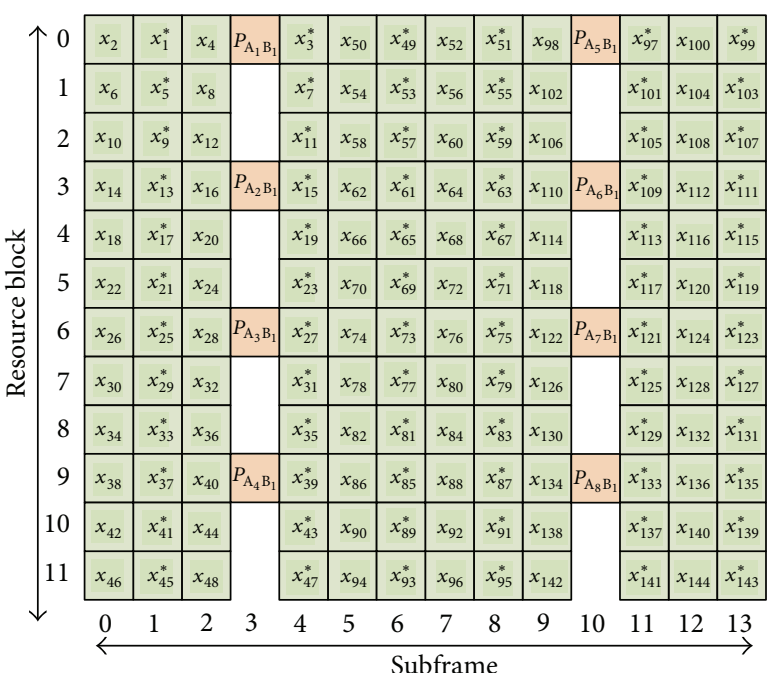

(a) Data mapping at $\operatorname{TxA}_{p} \mathrm{~B}_{1}$

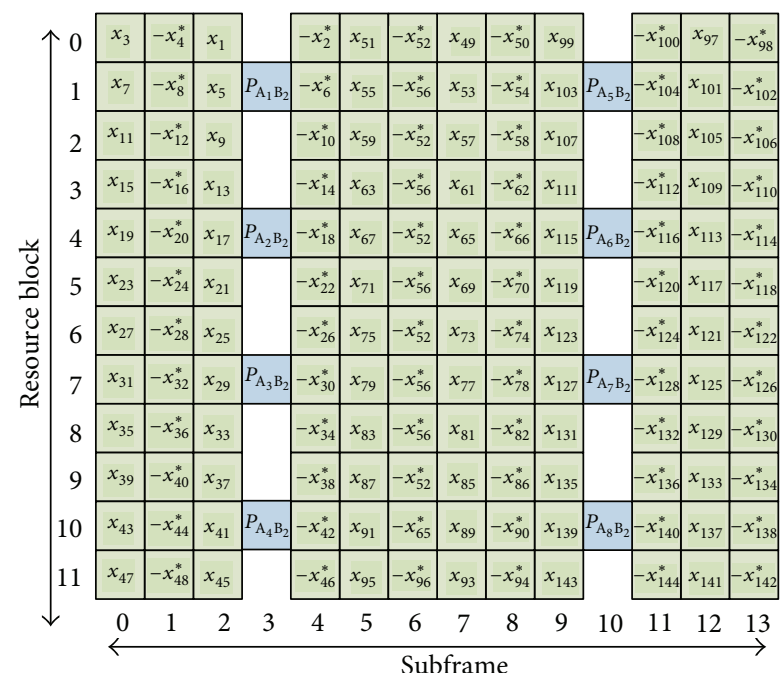

(b) Data mapping at $\mathrm{TxA}_{p} \mathrm{~B}_{2}$

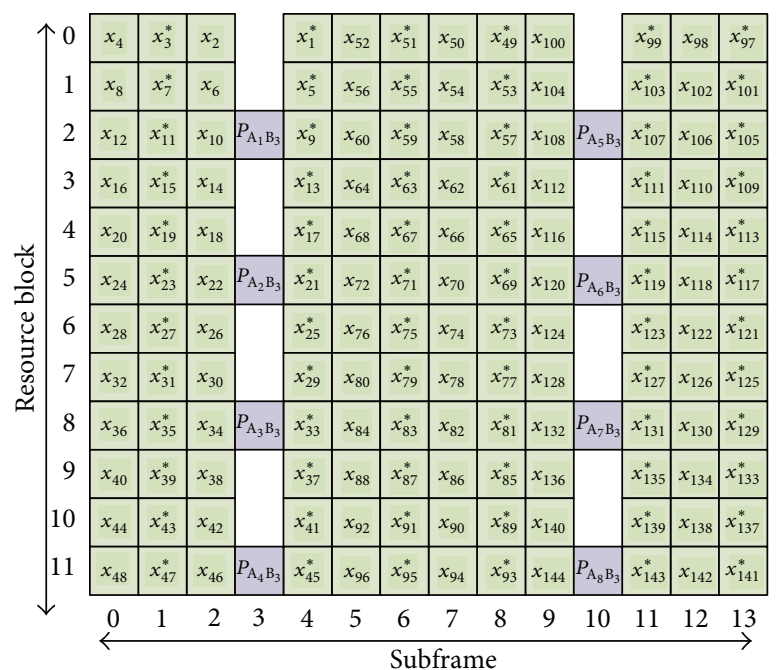

(c) Data mapping at $\mathrm{TxA}_{p} \mathrm{~B}_{3}$

FIGURE 5: Downlink data mapping of the LTE-A resource blocks for the TPULA system. 
TABLE 1: Simulation parameters.

\begin{tabular}{lc}
\hline System parameter & Value \\
\hline Carrier frequency & $1.8 \mathrm{GHz}$ \\
System bandwidth & $20 \mathrm{MHz}$ \\
FFT size & 2048 \\
Number of data carriers & 1200 \\
Number of samples in CP & 144 \\
Subcarrier spacing & $15 \mathrm{KHz}$ \\
Modulation & QPSK \\
Channel coding & $\mathrm{No}$ \\
MIMO configuration & 3B(Tx) by $2 \mathrm{~A} * 3 \mathrm{~B}(\mathrm{Rx})$ \\
Array spacing & Half-wavelength \\
\hline Channel parameter & Value \\
\hline & Indoor: 64, \\
Number of scatterers & Outdoor: 4 clusters with 16 \\
& scatterers per cluster $(64$ in total $)$ \\
Scatterer radius & $10 \mathrm{~m}$ \\
Rx velocity & $3 \mathrm{~km} / \mathrm{h}$ for indoor; \\
Fading & $100 \mathrm{~km} / \mathrm{h}$ for outdoor \\
XPD & Flat \\
Practical channel estimation & $5.8 \mathrm{~dB}$ \\
\hline
\end{tabular}

to separate the total number of AEs and only four AEs are dedicated to a mobile user. For example, there is about $5 \mathrm{~dB}$ SNR loss at target BLER in case of employing two users compared with the single user case. Meanwhile, Figure 9 demonstrates the simulated throughput curves for two users with the transmission Case 4, where system throughput is increased a lot when two users are employed. From Figure 9, we can observe that the single user cases have a higher throughput than the multiusers cases with a low SNR, for example, $-10 \mathrm{~dB}$, mainly due to a lower BLER. By increasing the SNR, the performance of multiusers gets better than the single user cases. This is because the proposed scheme is good at keeping the system BLER performance. When multiusers are employed, the system throughput then can be guaranteed to be linearly enhanced with the number of users being increased.

With the consideration of tradeoff regarding the spectrum efficiency, the blind channel estimation (BCE) [10], which requires no or a minimal number of pilots, might be better employed in the TPULA system in our future research. By using BCE, not only the system spectrum efficiency can be increased but also the error floor caused by the high mobility might be solved.

\section{Conclusions and Future Works}

This paper proposes a TPULA system associated with STBC and $\mathrm{BF}$ techniques to exploit diversity gain under a transmission scenario with both low and high correlated links. The polarized antenna branches are grouped into three sets, which steer three orthogonal beams at the $X-Y, Z-X$, and $Y-Z$

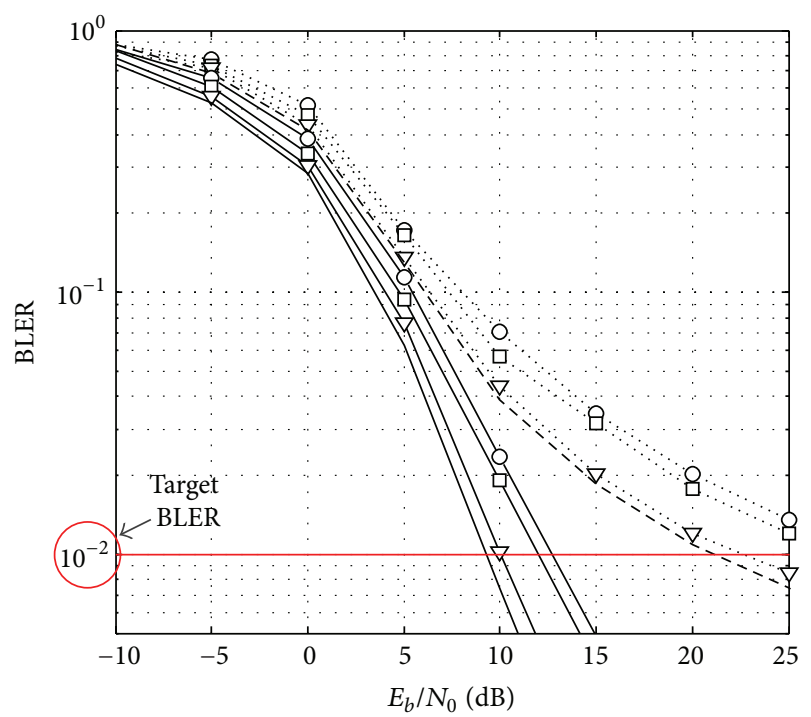

. O.. TPULA only, outdoor

口.. TPULA w/ STBC, outdoor

$\cdot \nabla \cdot$ TPULA w/ STBC and TxBF, outdoor

- - - TPULA w/ STBC and TRxBF, outdoor

-O- TPULA only, indoor

$\neg-$ TPULA w/ STBC, indoor

$\rightarrow-$ TPULA w/ STBC and TxBF, indoor

— TPULA w/ STBC and TRxBF, indoor

FIGURE 6: Simulation results of BLER for single user.

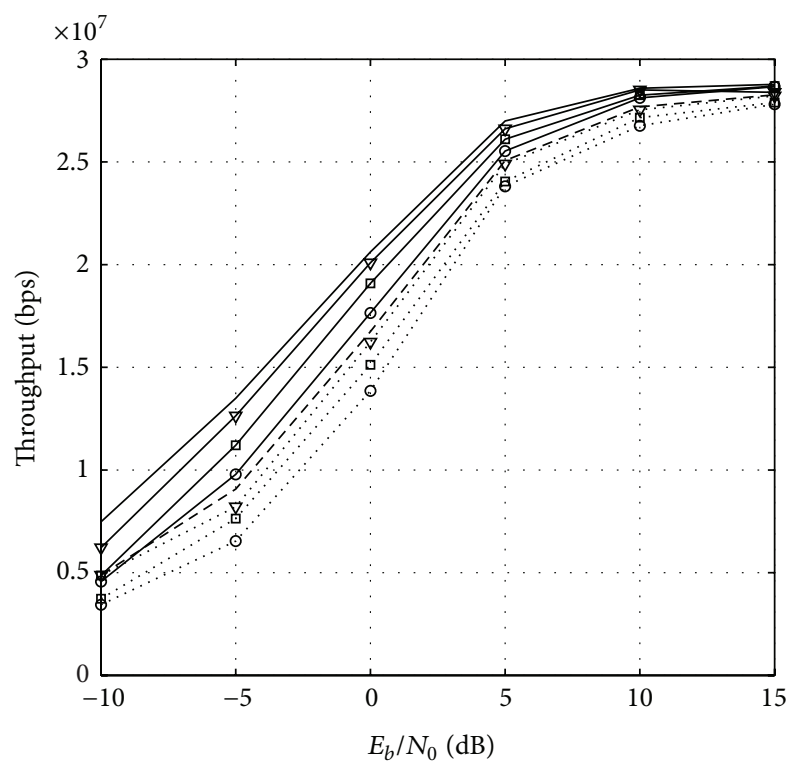

\footnotetext{
_ TPULA w/ STBC and TRxBF, indoor

$\checkmark$ TPULA w/ STBC and TxBF, indoor

$\square$ TPULA w/ STBC, indoor

$\checkmark$ TPULA only, indoor

- - - TPULA w/ STBC and TRxBF, outdoor

$\cdot \nabla \cdot$ TPULA w/ STBC and TxBF, outdoor

曰.. TPULA w/ STBC, outdoor

๑.. TPULA only, outdoor
}

Figure 7: Simulation results of throughput for single user. 


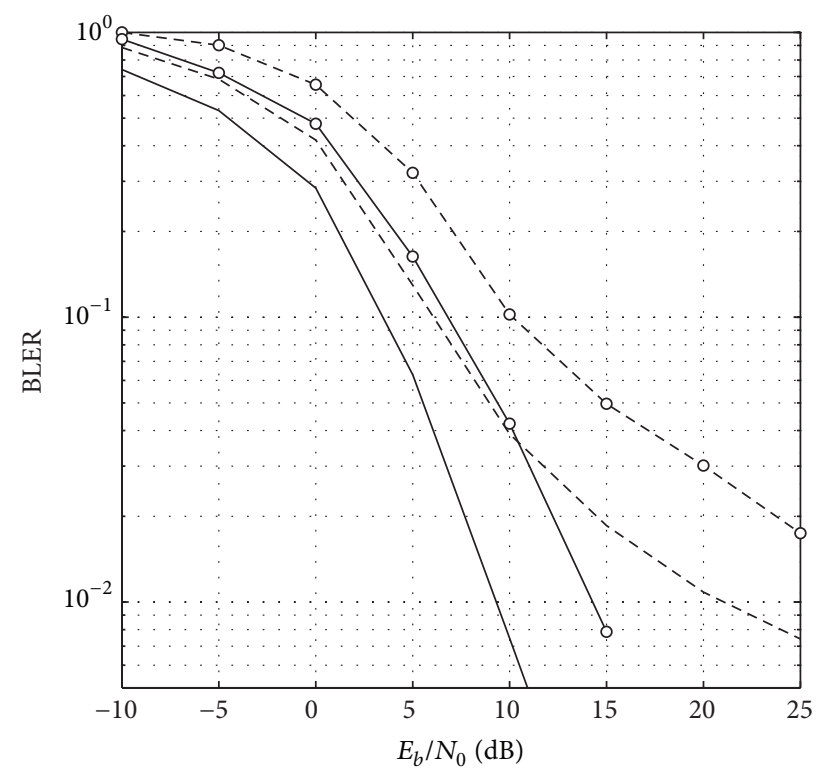

- o- TPULA w/ STBC and TRxBF, outdoor, two users

- - TPULA w/ STBC and TRxBF, outdoor, single user

- - TPULA w/ STBC and TRxBF, indoor, two users

— TPULA w/ STBC and TRxBF, indoor, single user

FIGURE 8: Simulation results of average BLER for two users.

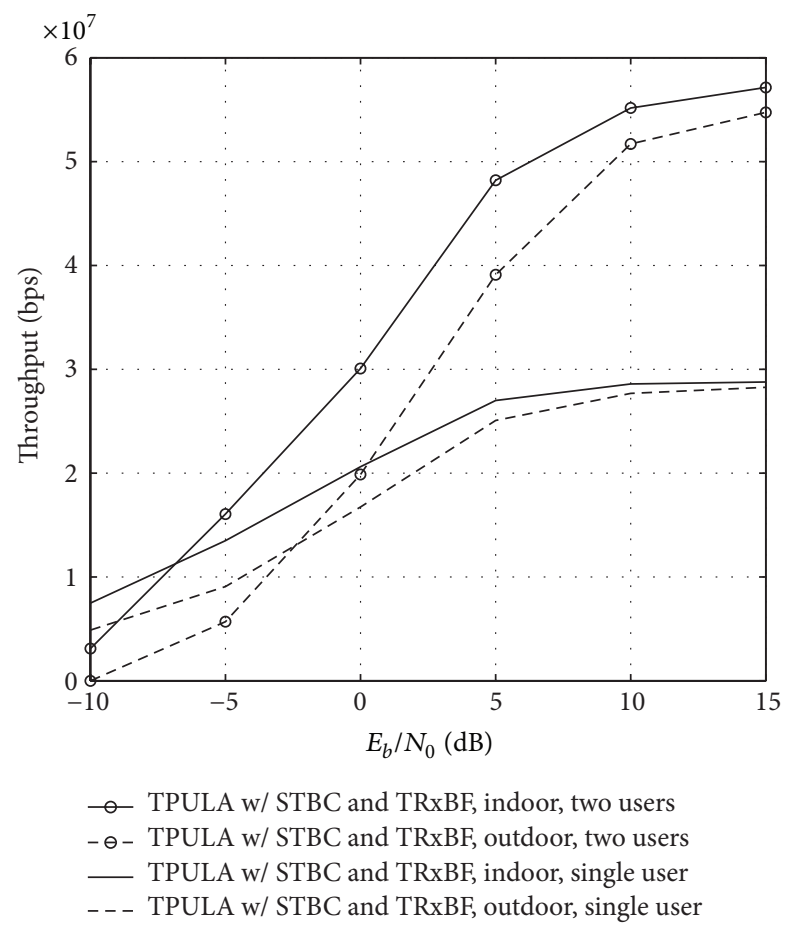

FIGURE 9: Simulation results of throughput for two users.

planes. STBC is additionally applied at three orthogonally colocated branches of each $\mathrm{AE}$ to accommodate the spacetime diversity gain. From the simulation results, the proposed scheme in Case 4 (TPULA with SBTC and TRxBF) proves we can obtain about $4 \mathrm{~dB}$ SNR gain at the target BLER compared with Case 1 (TPULA without STBC and TRxBF) under the indoor environment. Although the performance in an outdoor environment is also increased by the proposed scheme, the error-floors occur due to the high Rx mobility. In this paper, we have verified the proposed scheme under the flat fading environment only. As a future work, the selective fading environment will be examined, and the AE selection scheme for OFDM-based TPULA system might be proposed to overcome the ISI caused by multipath.

\section{Conflict of Interests}

The authors declare that there is no conflict of interests regarding the publication of this paper.

\section{Acknowledgment}

This research was supported by the MSIP (Ministry of Science, ICT and Future Planning), Korea, under the ITRC (Information Technology Research Center) Support Program (IITP-2015-H8501-15-1019) supervised by the IITP (Institute for Information \& Communications Technology Promotion).

\section{References}

[1] M.-T. Dao, V.-A. Nguyen, Y.-T. Im, S.-O. Park, and G. Yoon, "3D polarized channel modeling and performance comparison of MIMO antenna configurations with different polarizations," IEEE Transactions on Antennas and Propagation, vol. 59, no. 7, pp. 2672-2682, 2011.

[2] A. S. Y. Poon and D. N. C. Tse, "Degree-of-freedom gain from using polarimetric antenna elements," Institute of Electrical and Electronics Engineers. Transactions on Information Theory, vol. 57, no. 9, pp. 5695-5709, 2011.

[3] X. Su and K. H. Chang, "Polarized uniform linear array system: beam radiation pattern, beamforming diversity order, and channel capacity," International Journal of Antennas and Propagation, vol. 2015, Article ID 371236, 9 pages, 2015.

[4] J. Li, U. Park, and S. Y. Kim, "An efficient rate one STBC scheme with 3 transmit antennas," in Proceedings of the International Conference on Wireless Communications, Networking and Mobile Computing (WiCOM '08), pp. 1-4, IEEE, Dalian, China, October 2008.

[5] U. Park, S. Kim, K. Lim, and J. Li, "A novel QO-STBC scheme with linear decoding for three and four transmit antennas," IEEE Communications Letters, vol. 12, no. 12, pp. 868-870, 2008.

[6] V. Madisetti, Digital Signal Processing Handbook, CRC Press, 2nd edition, 1999.

[7] L. J. Liu, S. Y. Kim, and M.-S. Lim, "An efficient selective receiver for STBC scheme," in Proceedings of the IEEE International Conference on Communications (ICC '07), pp. 4196-4200, IEEE, Glasgow, Scotland, June 2007.

[8] W. Liu, "Adaptive wideband beamforming with sensor delaylines," Signal Processing, vol. 89, no. 5, pp. 876-882, 2009.

[9] X. Su, B. Hui, and K. H. Chang, "3-D MIMO channel modeling with beamforming analysis for dual-polarized antenna 
systems," in Proceedings of the IEEE 78th Vehicular Technology Conference (VTC Fall '13), pp. 1-5, September 2013.

[10] E. Beres and R. Adve, "Blind channel estimation for orthogonal STBC in MISO systems," IEEE Transactions on Vehicular Technology, vol. 56, no. 4, pp. 2042-2050, 2007. 

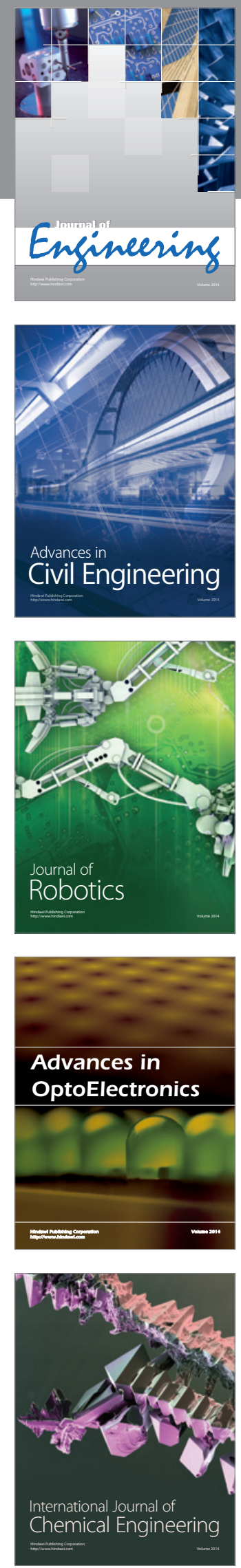

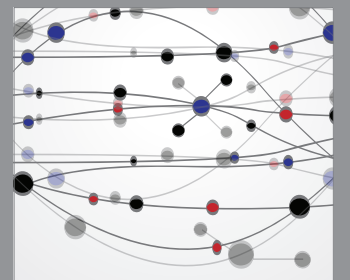

The Scientific World Journal
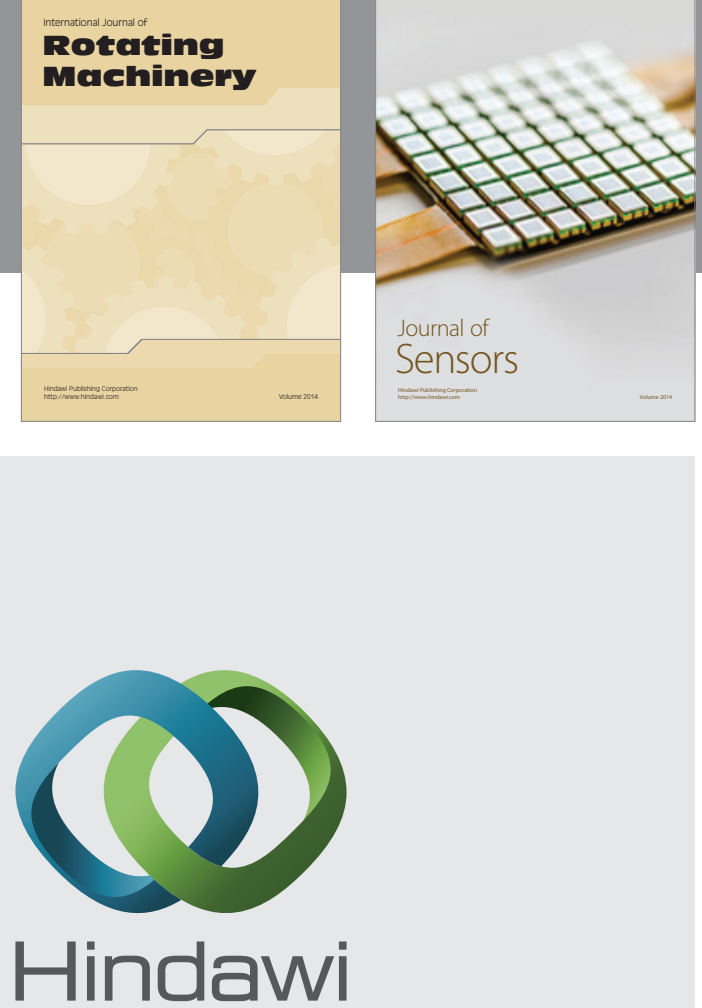

Submit your manuscripts at http://www.hindawi.com
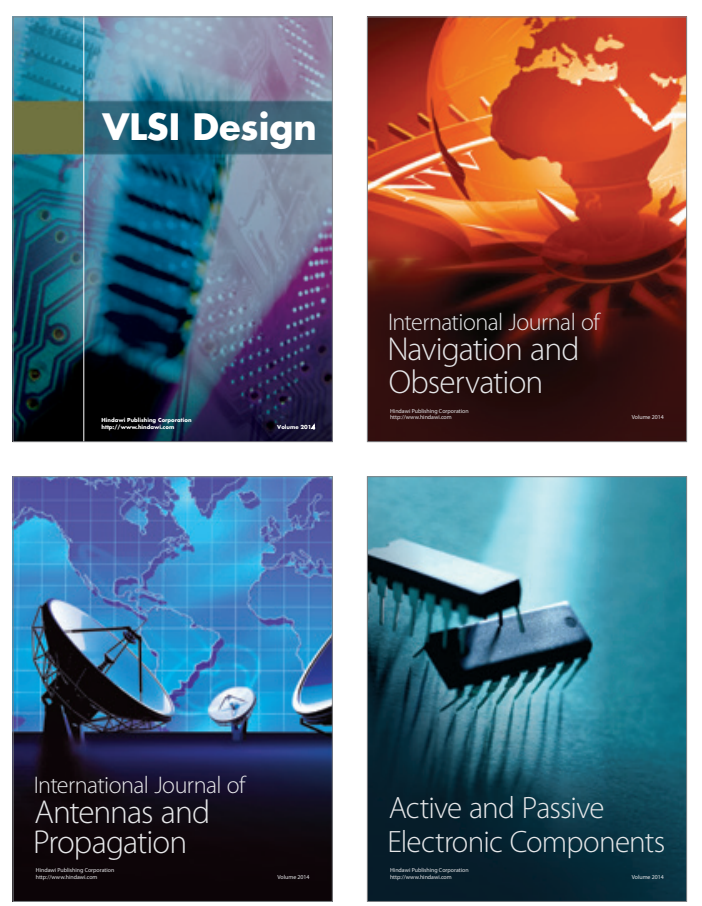
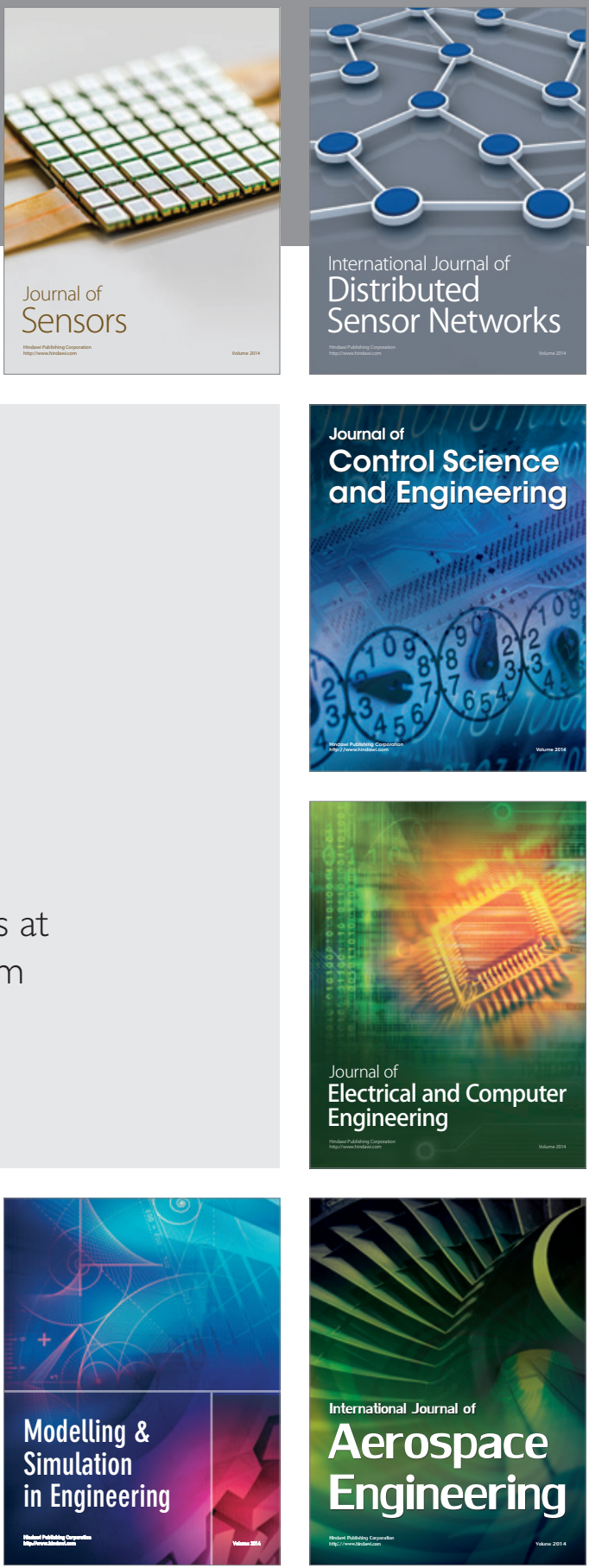

Journal of

Control Science

and Engineering
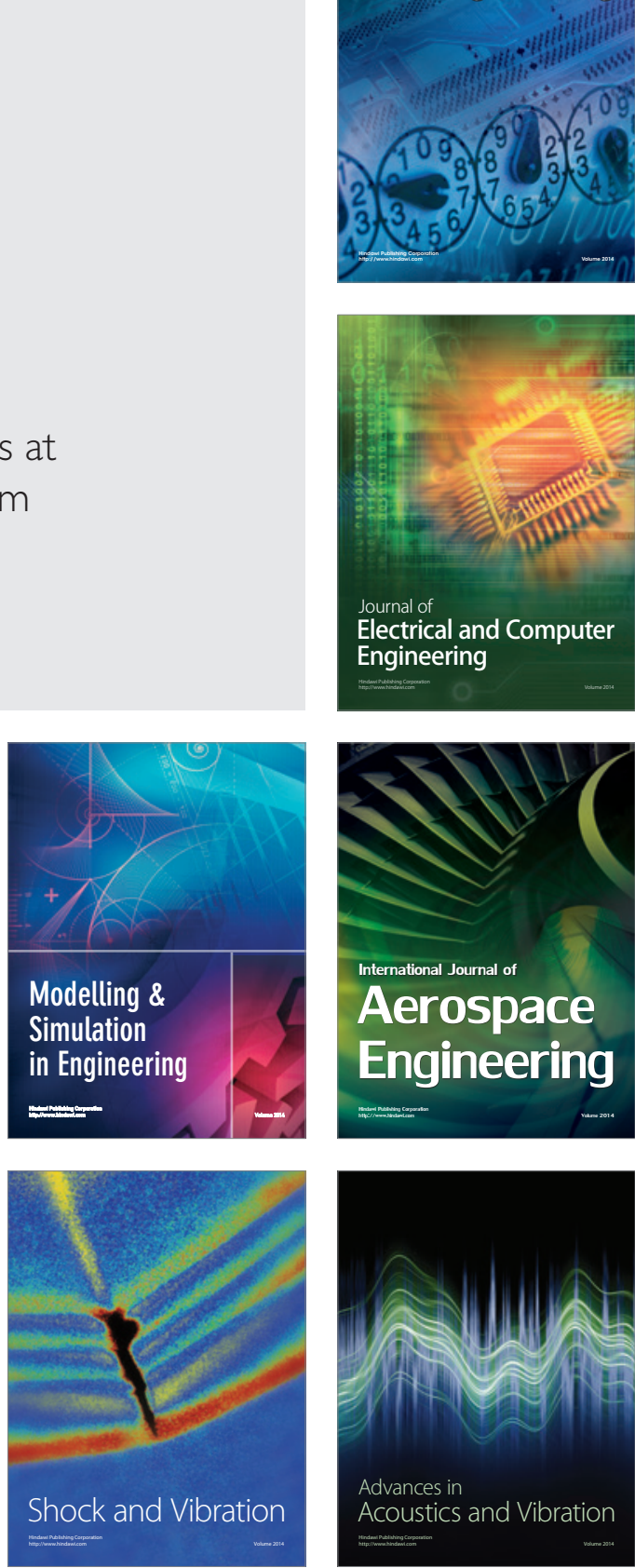